\title{
Optimal dynamic reinsurance with dependent risks: variance premium principle
}

\author{
Zhibin Liang ${ }^{*}$ Kam Chuen Yuen ${ }^{2}$ \\ ${ }^{1}$ School of Mathematical Sciences, Nanjing Normal University, \\ Jiangsu 210023, P.R.China \\ ${ }^{2}$ Department of Statistics and Actuarial Science, \\ The University of Hong Kong, Pokfulam Road, Hong Kong
}

${ }^{*}$ Corresponding author: Dr. Zhibin Liang, Email: liangzhibin111@hotmail.com 


\begin{abstract}
In this paper, we consider the optimal proportional reinsurance strategy in a risk model with two dependent classes of insurance business, where the two claim number processes are correlated through a common shock component. Under the criterion of maximizing the expected exponential utility with the variance premium principle, we adopt a nonstandard approach to examining the existence and uniqueness of the optimal reinsurance strategy. Using the technique of stochastic control theory, closed-form expressions for the optimal strategy and the value function are derived for the compound Poisson risk model as well as for the Brownian motion risk model. From the numerical examples, we see that the optimal results for the compound Poisson risk model are very different from those for the diffusion model. The former depends not only on the safety loading, time, and the interest rate, but also on the claim size distributions and the claim number processes, while the latter only depends on the safety loading, time, and the interest rate.
\end{abstract}

Keywords: Brownian motion; Common shock; Compound Poisson process; Diffusion process; Exponential utility; Hamilton-Jacobi-Bellman equation; Proportional reinsurance 


\section{Introduction}

With reinsurance, insurers are able to transfer some of their risks to another party at the expense of making less potential profit, and hence finding optimal reinsurance strategy to balance their risk and profit is of great interest to them. In fact, optimal reinsurance problems have gained much interest in the actuarial literature in the past few years, and the technique of stochastic control theory and the Hamilton-Jacobi-Bellman equation are frequently used to cope with these problems. See, for example, Schmidli (2001), Irgens and Paulsen (2004), Promislow and Young (2005), Liang et al. (2011), and Liang and Young (2012).

In the study of optimal reinsurance contracts, a few objective functions are commonly seen in the literature. Browne (1995), Schmidli (2002), Liang (2007), and Luo et al. (2008) consider the objective function that minimizes ruin probability. Kaluszka $(2001,2004)$ study the optimal reinsurance problem under various mean-variance premium principles of the reinsurer. Since explicit expression for the ruin probability is difficult to derive when the underlying risk follows a compound Poisson process, some papers including Centeno (1986, 2002), Hald and Schmidli (2004), and Liang and Guo $(2007,2008)$ focus on constructing optimal contracts that maximize the adjustment coefficient by the martingale approach. Moreover, Cai and Tan (2007), Cai et al. (2008), and Bernard and Tian (2009) adopt the criteria of minimizing tail risk measures such as value at risk and conditional tail expectation. In this paper, our objective is to maximize the expected utility of terminal wealth which is another popular criterion for various optimization problems in finance and modern risk theory. For example, see Liang et al. (2012) and references therein.

Although research on optimal reinsurance is increasing rapidly, only a few papers deal with the problem in relation to dependent risks. Under the criteria of maximizing the expected utility of terminal wealth and maximizing the adjustment coefficient, Centeno (2005) studies the optimal excess of loss retention limits for two dependent classes of insurance risks. Bai et al. (2012) also seek the optimal excess of loss reinsurance to minimize the ruin probability for the diffusion risk model. Under the variance premium principle, the optimal reinsurance contract is not necessarily an excess of loss reinsurance but a proportional reinsurance (see Proposition 7 in Hipp and Taksar (2010)). These papers motivate us to consider the optimal proportional reinsurance with dependent risks under the variance premium principle. By a nonstandard approach, we investigate the conditions

of existence and uniqueness of the optimal reinsurance strategies. Using the technique of stochastic control theory, closed-form expressions for the optimal reinsurance strategy and the value function 
are derived for the compound Poisson risk model as well as for the diffusion risk model. From the numerical examples, we find that the optimal results in the compound Poisson case are very different from those in the diffusion case. The former depends not only on the safety loading, time, and interest rate, but also on the claim size distributions and the counting processes, while the latter only depends on the safety loading, time, and interest rate.

The rest of the paper is organized as follows. In Section 2, the models and assumptions are presented. In Sections 3 and 4, we discuss the optimal strategies in both the compound Poisson and diffusion cases, and derive closed form expressions for the optimal results. In Section 5, numerical examples are carried out to assess the impact of some model parameters on the optimal strategies. Finally, we conclude the paper in Section 6.

\section{Model formulation}

Suppose that an insurance company has two dependent classes of insurance business such as motor, health, and life insurance. Let $X_{i}$ be the claim size random variables for the first class with common distribution $F_{X}(x)$ and $Y_{i}$ be the claim size random variables for the second class with common distribution $F_{Y}(y)$. Their means are denoted by $\mu_{1}=E\left(X_{i}\right)$ and $\mu_{2}=E\left(Y_{i}\right)$. Assume that $F_{X}(x)=0$ for $x \leq 0, F_{Y}(y)=0$ for $y \leq 0,0<F_{X}(x)<1$ for $x>0,0<F_{Y}(y)<1$ for $y>0$, and that their moment generating functions, $M_{X}(r)$ and $M_{Y}(r)$, exist. Then, the aggregate claims processes for the two classes are given by

$$
S_{1}(t)=\sum_{i=1}^{M_{1}(t)} X_{i} \quad \text { and } \quad S_{2}(t)=\sum_{i=1}^{M_{2}(t)} Y_{i},
$$

where $M_{i}(t)$ is the claim number process for class $i(i=1,2)$. It is assumed that $X_{i}$ and $Y_{i}$ are independent claim size random variables, and that they are independent of $M_{1}(t)$ and $M_{2}(t)$.

The two claim number processes are correlated in the way that

$$
M_{1}(t)=N_{1}(t)+N(t) \quad \text { and } \quad M_{2}(t)=N_{2}(t)+N(t),
$$

with $N_{1}(t), N_{2}(t)$, and $N(t)$ being three independent Poisson processes with parameters $\lambda_{1}, \lambda_{2}$, and $\lambda$, respectively. Therefore, the aggregate claims process generated from the two classes of business has the form

$$
S_{t}=\sum_{i=1}^{N_{1}(t)+N(t)} X_{i}+\sum_{i=1}^{N_{2}(t)+N(t)} Y_{i}
$$


We assume that both $E\left(X e^{r X}\right)=M_{X}^{\prime}(r)$ and $E\left(Y e^{r Y}\right)=M_{Y}^{\prime}(r)$ exist for $0<r<\zeta$, and that both $\lim _{r \rightarrow \zeta} E\left(X e^{r X}\right)$ and $\lim _{r \rightarrow \zeta} E\left(Y e^{r Y}\right)$ tend to $\infty$ for some $0<\zeta \leq+\infty$. It is obvious that the dependence of the two classes of business is due to a common shock governed by the counting process $N(t)$. This model has been studied extensively in the literature; see for example, Yuen et al. $(2002,2006)$.

As usual, we define the surplus process

$$
R_{t}=u+c t-S_{t}
$$

where $u$ is the amount of initial surplus, and $c$ is the rate of premium. Moreover, we allow the insurance company to continuously reinsure a fraction of its claim with the retention levels $q_{1 t} \in$ $[0,1]$ and $q_{2 t} \in[0,1]$ for $X_{i}$ and $Y_{i}$, respectively. Let the reinsurance premium rate at time $t$ be $\delta\left(q_{1 t}, q_{2 t}\right)$. Furthermore, the company is allowed to invest all its surplus in a risk free asset with interest rate $r$. Let $\left\{R_{t}^{q_{1}, q_{2}}, t \geq 0\right\}$ denote the associated surplus process, i.e., $R_{t}^{q_{1}, q_{2}}$ is the wealth of the insurer at time $t$ under the strategy $\left(q_{1 t}, q_{2 t}\right)$. This process then evolves as

$$
d R_{t}^{q_{1}, q_{2}}=\left[r R_{t}^{q_{1}, q_{2}}+\left(c-\delta\left(q_{1 t}, q_{2 t}\right)\right)\right] d t-q_{1 t} d S_{1}(t)-q_{2 t} d S_{2}(t)
$$

From Grandell (1991), we know that the Brownian motion risk model given by

$$
\hat{S}_{1}(t)=a_{1} t-\sigma_{1} B_{1 t},
$$

with $a_{1}=\left(\lambda_{1}+\lambda\right) E(X)$ and $\sigma_{1}^{2}=\left(\lambda_{1}+\lambda\right) E\left(X^{2}\right)$ can be seen as a diffusion approximation to the compound Poisson process $S_{1}(t)$. Similarly,

$$
\hat{S}_{2}(t)=a_{2} t-\sigma_{2} B_{2 t},
$$

with $a_{2}=\left(\lambda_{2}+\lambda\right) E(Y)$ and $\sigma_{2}^{2}=\left(\lambda_{2}+\lambda\right) E\left(Y^{2}\right)$ can be treated as a diffusion approximation to the compound Poisson process $S_{2}(t)$. Here, $B_{1 t}$ and $B_{2 t}$ are standard Brownian motions with the correlation coefficient

$$
\rho=\frac{\lambda E(X) E(Y)}{\sqrt{\left(\lambda_{1}+\lambda\right) E\left(X^{2}\right)\left(\lambda_{2}+\lambda\right) E\left(Y^{2}\right)}},
$$

So, $E\left[B_{1 t} B_{2 t}\right]=\rho t$. Replacing $S_{i}(t)(i=1,2)$ of $(2.1)$ by $\hat{S}_{i}(t)(i=1,2)$, one can obtain the following surplus process

$$
d \hat{R}_{t}^{q_{1}, q_{2}}=\left[r \hat{R}_{t}^{q_{1}, q_{2}}+\left(c-\delta\left(q_{1 t}, q_{2 t}\right)\right)-q_{1 t} a_{1}-q_{2 t} a_{2}\right] d t+q_{1 t} \sigma_{1} d B_{1 t}+q_{2 t} \sigma_{2} d B_{2 t},
$$


or equivalently,

$$
\begin{aligned}
d \hat{R}_{t}^{q_{1}, q_{2}}= & {\left[r \hat{R}_{t}^{q_{1}, q_{2}}+\left(c-\delta\left(q_{1 t}, q_{2 t}\right)\right)-q_{1 t} a_{1}-q_{2 t} a_{2}\right] d t } \\
& +\sqrt{\sigma_{1}^{2} q_{1 t}^{2}+\sigma_{2}^{2} q_{2 t}^{2}+2 \sigma_{1} \sigma_{2} q_{1 t} q_{2 t} \rho} d B_{t}, \\
= & {\left[r \hat{R}_{t}^{q_{1}, q_{2}}+\left(c-\delta\left(q_{1 t}, q_{2 t}\right)\right)-q_{1 t} a_{1}-q_{2 t} a_{2}\right] d t } \\
& +\sqrt{\sigma_{1}^{2} q_{1 t}^{2}+\sigma_{2}^{2} q_{2 t}^{2}+2 q_{1 t} q_{2 t} \lambda \mu_{1} \mu_{2}} d B_{t},
\end{aligned}
$$

where $B_{t}$ is a standard Brownian motion.

Remark 2.1. It follows from Yuen et al. (2002) or Wang and Yuen (2005) that $S_{t}$ is also a compound Poisson process with parameter $\tilde{\lambda}=\lambda_{1}+\lambda_{2}+\lambda$, and that the distribution of the transformed claim size random variable $X^{\prime}$ is given by

$$
F_{X^{\prime}}(x)=\frac{\lambda_{1}}{\tilde{\lambda}} F_{X}(x)+\frac{\lambda_{2}}{\tilde{\lambda}} F_{Y}(x)+\frac{\lambda}{\tilde{\lambda}} F_{X+Y}(x) .
$$

Therefore, the Brownian motion risk model given by

$$
\hat{S}_{t}=\left(a_{1}+a_{2}\right) t-\sqrt{\sigma_{1}^{2}+\sigma_{2}^{2}+2 \lambda \mu_{1} \mu_{2}} B_{t}
$$

can be seen as a diffusion approximation to the compound Poisson process $S_{t}$. On the other hand,

$$
\hat{S}_{1}(t)+\hat{S}_{2}(t)=\left(a_{1}+a_{2}\right) t-\left(\sigma_{1} B_{1 t}+\sigma_{2} B_{2 t}\right),
$$

which can be replaced by

$$
\hat{S}_{1}(t)+\hat{S}_{2}(t)=\left(a_{1}+a_{2}\right) t-\sqrt{\sigma_{1}^{2}+\sigma_{2}^{2}+2 \sigma_{1} \sigma_{2} \rho} B_{t}
$$

as the two forms have the same distributional properties. Hence, the sum $\hat{S}_{1}(t)+\hat{S}_{2}(t)$ can also be regarded as a diffusion approximation to the compound Poisson process $S_{t}$ when

$$
\rho=\frac{\lambda E(X) E(Y)}{\sqrt{\left(\lambda_{1}+\lambda\right) E\left(X^{2}\right)\left(\lambda_{2}+\lambda\right) E\left(Y^{2}\right)}}=\frac{\lambda \mu_{1} \mu_{2}}{\sigma_{1} \sigma_{2}} .
$$

Assume now that the insurer is interested in maximizing the expected utility of terminal wealth, say at time $T$. The utility function is $u(x)$, which satisfies $u^{\prime}>0$ and $u^{\prime \prime}<0$. Then, the objective functions are

$$
J^{q_{1}, q_{2}}(t, x)=E\left[u\left(R_{T}^{q_{1}, q_{2}}\right) \mid R_{t}^{q_{1}, q_{2}}=x\right],
$$

and

$$
J^{q_{1}, q_{2}}(t, x)=E\left[u\left(\hat{R}_{T}^{q_{1}, q_{2}}\right) \mid \hat{R}_{t}^{q_{1}, q_{2}}=x\right] .
$$


Since (2.3) and (2.4) will be discussed separately, the use of the same notation $J^{q_{1}, q_{2}}(t, x)$ will not cause any confusion. The corresponding value function is then given by

$$
V(t, x)=\sup _{q_{1}, q_{2}} J^{q_{1}, q_{2}}(t, x) .
$$

We assume that the insurer has an exponential utility function

$$
u(x)=-\frac{m}{\nu} e^{-\nu x}
$$

for $m>0$ and $\nu>0$. This utility has constant absolute risk aversion (CARA) parameter $\nu$. Such a utility function plays an important role in insurance mathematics and actuarial practice as this is the only function under which the principle of "zero utility" gives a fair premium that is independent of the level of reserve of an insurance company (see Gerber (1979)).

Let $C^{1,2}$ denote the space of $\phi(t, x)$ such that $\phi$ and its partial derivatives $\phi_{t}, \phi_{x}, \phi_{x x}$ are continuous on $[0, T] \times R$. To solve the above problem, we use the dynamic programming approach described in Fleming and Soner (2006). From the standard arguments, we see that if the value function $V \in C^{1,2}$, then $V$ satisfies the following Hamilton-Jacobi-Bellman (HJB) equation

$$
\sup _{q_{1}, q_{2}} \mathcal{A}^{q_{1}, q_{2}} V(t, x)=0
$$

for $t<T$ with the boundary condition

$$
V(T, x)=u(x)
$$

where

$$
\begin{aligned}
\mathcal{A}^{q_{1}, q_{2}} V(t, x)=V_{t} & +\left[r x+c-\delta\left(q_{1}, q_{2}\right)\right] V_{x} \\
& +\lambda_{1} E\left[V\left(t, x-q_{1} X\right)-V(t, x)\right] \\
& +\lambda_{2} E\left[V\left(t, x-q_{2} Y\right)-V(t, x)\right] \\
& +\lambda E\left[V\left(t, x-q_{1} X-q_{2} Y\right)-V(t, x)\right],
\end{aligned}
$$

for the risk process $(2.1)$, and

$$
\begin{aligned}
\mathcal{A}^{q_{1}, q_{2}} V(t, x)=V_{t} & +\left[r x+c-\delta\left(q_{1}, q_{2}\right)-q_{1} a_{1}-q_{2} a_{2}\right] V_{x} \\
& +\frac{1}{2}\left(\sigma_{1}^{2} q_{1}^{2}+\sigma_{2}^{2} q_{2}^{2}+2 q_{1} q_{2} \lambda \mu_{1} \mu_{2}\right) V_{x x}
\end{aligned}
$$

for the risk process $(2.2)$.

Using the standard methods of Fleming and Soner (2006) and Yang and Zhang (2005), we have the following verification theorem: 
Theorem 2.1. Let $W \in C^{1,2}$ be a classical solution to (2.6) that satisfies (2.7). Then, the value function $V$ given by (2.5) coincides with $W$. That is,

$$
W(t, x)=V(t, x)
$$

Furthermore, set $\left(q_{1}^{*}, q_{2}^{*}\right)$ such that

$$
\mathcal{A}^{q_{1}^{*}, q_{2}^{*}} V(t, x)=0
$$

holds for all $(t, x) \in[0, T) \times R$. Then, $\left(q_{1}^{*}\left(t, R_{t}^{*}\right), q_{2}^{*}\left(t, R_{t}^{*}\right)\right)$ is the optimal strategy. Here, $R_{t}^{*}$ is the surplus process under the optimal strategy.

Remark 2.2. In this paper, we assume that continuous trading is allowed and that all assets are infinitely divisible. We work on a complete probability space $(\Omega, \mathcal{F}, P)$ on which the process $R_{t}^{q_{1}, q_{2}}$ is well defined. The information at time $t$ is given by the complete filtration $\mathcal{F}_{t}$ generated by $R_{t}^{q_{1}, q_{2}}$. The strategy $\left(q_{1 t}, q_{2 t}\right)$ is $\mathcal{F}_{t}$-predictable.

\section{Optimal results for the compound Poisson model}

In this section, we consider the optimization problem for the risk model (2.1). Throughout the paper, we assume that the reinsurance premium is calculated according to the variance principle. That is,

$$
\delta\left(q_{1}, q_{2}\right)=\left(1-q_{1}\right) a_{1}+\left(1-q_{2}\right) a_{2}+\Lambda \tilde{h}\left(q_{1}, q_{2}\right) .
$$

Here, $\Lambda>0$ is the safety loading of the reinsurer, and

$$
\tilde{h}\left(q_{1}, q_{2}\right)=\left(1-q_{1}\right)^{2} \sigma_{1}^{2}+\left(1-q_{2}\right)^{2} \sigma_{2}^{2}+2\left(1-q_{1}\right)\left(1-q_{2}\right) \lambda \mu_{1} \mu_{2} .
$$

To solve the equation

$$
\begin{aligned}
\sup _{q_{1}, q_{2}}\{ & V_{t}+\left[r x+c-\delta\left(q_{1}, q_{2}\right)\right] V_{x}+\lambda_{1} E\left[V\left(t, x-q_{1} X\right)-V(t, x)\right] \\
& \left.+\lambda_{2} E\left[V\left(t, x-q_{2} Y\right)-V(t, x)\right]+\lambda E\left[V\left(t, x-q_{1} X-q_{2} Y\right)-V(t, x)\right]\right\}=0,
\end{aligned}
$$

with the boundary condition $V(T, x)=u(x)$, inspired by Browne (1995), we try to fit a solution with the form

$$
V(t, x)=-\frac{m}{\nu} \exp \left[-\nu x e^{r(T-t)}+h(T-t)\right]
$$

where $h(\cdot)$ is a suitable function such that (3.2) is a solution to (2.6). The boundary condition $V(T, x)=u(x)$ implies that $h(0)=0$. 
From (3.2), we get

$$
\left\{\begin{array}{l}
V_{t}=V(t, x)\left[\nu x r e^{r(T-t)}-h^{\prime}(T-t)\right], \\
V_{x}=V(t, x)\left[-\nu e^{r(T-t)}\right], \\
V_{x x}=V(t, x)\left[\nu^{2} e^{2 r(T-t)}\right], \\
E\left[V\left(t, x-q_{1} X\right)-V(t, x)\right]=V(t, x)\left[M_{X}\left(\nu q_{1} e^{r(T-t)}\right)-1\right], \\
E\left[V\left(t, x-q_{2} Y\right)-V(t, x)\right]=V(t, x)\left[M_{Y}\left(\nu q_{2} e^{r(T-t)}\right)-1\right], \\
E\left[V\left(t, x-q_{1} X-q_{2} Y\right)-V(t, x)\right] \\
=V(t, x)\left[M_{X}\left(\nu q_{1} e^{r(T-t)}\right) M_{Y}\left(\nu q_{2} e^{r(T-t)}\right)-1\right] .
\end{array}\right.
$$

Putting (3.3) into the equation (2.6) and rearranging terms yield

$$
\begin{aligned}
\inf _{q_{1}, q_{2}}\{ & -h^{\prime}(T-t)-c \nu e^{r(T-t)}-\lambda_{1}-\lambda_{2}-\lambda+\delta\left(q_{1}, q_{2}\right) \nu e^{r(T-t)} \\
& +\lambda_{1} M_{X}\left(\nu q_{1} e^{r(T-t)}\right)+\lambda_{2} M_{Y}\left(\nu q_{2} e^{r(T-t)}\right) \\
& \left.+\lambda M_{X}\left(\nu q_{1} e^{r(T-t)}\right) M_{Y}\left(\nu q_{2} e^{r(T-t)}\right)\right\}=0
\end{aligned}
$$

for $t<T$. Let

$$
\begin{aligned}
\tilde{f}\left(q_{1}, q_{2}\right)= & \delta\left(q_{1}, q_{2}\right) \nu e^{r(T-t)}+\lambda_{1} M_{X}\left(\nu q_{1} e^{r(T-t)}\right) \\
& +\lambda_{2} M_{Y}\left(\nu q_{2} e^{r(T-t)}\right)+\lambda M_{X}\left(\nu q_{1} e^{r(T-t)}\right) M_{Y}\left(\nu q_{2} e^{r(T-t)}\right) .
\end{aligned}
$$

For any $t \in[0, T]$, we have

$$
\left\{\begin{array}{l}
\frac{\partial \tilde{f}\left(q_{1}, q_{2}\right)}{\partial q_{1}}=\left(\frac{\partial \delta\left(q_{1}, q_{2}\right)}{\partial q_{1}}+M_{X}^{\prime}\left(\nu q_{1} e^{r(T-t)}\right)\left(\lambda_{1}+\lambda M_{Y}\left(\nu q_{2} e^{r(T-t)}\right)\right)\right) \cdot \nu e^{r(T-t)} \\
\frac{\partial \tilde{f}\left(q_{1}, q_{2}\right)}{\partial q_{2}}=\left(\frac{\partial \delta\left(q_{1}, q_{2}\right)}{\partial q_{2}}+M_{Y}^{\prime}\left(\nu q_{2} e^{r(T-t)}\right)\left(\lambda_{2}+\lambda M_{X}\left(\nu q_{1} e^{r(T-t)}\right)\right)\right) \cdot \nu e^{r(T-t)}
\end{array}\right.
$$

Moreover, since

$$
\delta\left(q_{1}, q_{2}\right)=\left(1-q_{1}\right) a_{1}+\left(1-q_{2}\right) a_{2}+\Lambda \tilde{h}\left(q_{1}, q_{2}\right),
$$

we obtain

$$
\left\{\begin{aligned}
\frac{\partial \tilde{f}\left(q_{1}, q_{2}\right)}{\partial q_{1}}=( & a_{1}-\Lambda\left(2\left(1-q_{1}\right) \sigma_{1}^{2}+2\left(1-q_{2}\right) \lambda \mu_{1} \mu_{2}\right) \\
& \left.+M_{X}^{\prime}\left(\nu q_{1} e^{r(T-t)}\right)\left(\lambda_{1}+\lambda M_{Y}\left(\nu q_{2} e^{r(T-t)}\right)\right)\right) \cdot \nu e^{r(T-t)} \\
\frac{\partial \tilde{f}\left(q_{1}, q_{2}\right)}{\partial q_{2}}= & \left(-a_{2}-\Lambda\left(2\left(1-q_{2}\right) \sigma_{2}^{2}+2\left(1-q_{1}\right) \lambda \mu_{1} \mu_{2}\right)\right. \\
& \left.+M_{Y}^{\prime}\left(\nu q_{2} e^{r(T-t)}\right)\left(\lambda_{2}+\lambda M_{X}\left(\nu q_{1} e^{r(T-t)}\right)\right)\right) \cdot \nu e^{r(T-t)}
\end{aligned}\right.
$$


and

$$
\left\{\begin{aligned}
\frac{\partial^{2} \tilde{f}\left(q_{1}, q_{2}\right)}{\partial q_{1}^{2}}= & M_{X}^{\prime \prime}\left(\nu q_{1} e^{r(T-t)}\right)\left(\lambda_{1}+\lambda M_{Y}\left(\nu q_{2} e^{r(T-t)}\right)\right) \cdot \nu^{2} e^{2 r(T-t)} \\
& +2 \Lambda \sigma_{1}^{2} \cdot \nu e^{r(T-t)}>0 \\
\frac{\partial^{2} \tilde{f}\left(q_{1}, q_{2}\right)}{\partial q_{2}^{2}}= & M_{Y}^{\prime \prime}\left(\nu q_{2} e^{r(T-t)}\right)\left(\lambda_{2}+\lambda M_{X}\left(\nu q_{1} e^{r(T-t)}\right)\right) \cdot \nu^{2} e^{2 r(T-t)} \\
& +2 \Lambda \sigma_{2}^{2} \cdot \nu e^{r(T-t)}>0 \\
\frac{\partial^{2} \tilde{f}\left(q_{1}, q_{2}\right)}{\partial q_{1} \partial q_{2}}= & \frac{\partial^{2} \tilde{f}\left(q_{1}, q_{2}\right)}{\partial q_{2} \partial q_{1}}=2 \Lambda \lambda \mu_{1} \mu_{2} \nu e^{r(T-t)} \\
& \left.\quad+\lambda M_{X}^{\prime}\left(\nu q_{1} e^{r(T-t)}\right) \cdot M_{Y}^{\prime}\left(\nu q_{2} e^{r(T-t)}\right)\right) \cdot \nu^{2} e^{2 r(T-t)} .
\end{aligned}\right.
$$

Lemma 3.1. $\tilde{f}\left(q_{1}, q_{2}\right)$ is a convex function with respect to $q_{1}$ and $q_{2}$.

Proof: To prove $\tilde{f}\left(q_{1}, q_{2}\right)$ is a convex function with respect to $q_{1}$ and $q_{2}$, it is sufficient to prove that the Hessian matrix of $\tilde{f}\left(q_{1}, q_{2}\right)$ is positive definite.

Let

$$
\mathbf{A}=\left(\begin{array}{cc}
\sigma_{1}^{2} & \lambda \mu_{1} \mu_{2} \\
& \\
\lambda \mu_{1} \mu_{2} & \sigma_{2}^{2}
\end{array}\right), \quad \mathbf{B}=\left(\begin{array}{cc}
\lambda_{1} M_{X}^{\prime \prime}\left(\nu q_{1} e^{r(T-t)}\right) & 0 \\
0 & \lambda_{2} M_{Y}^{\prime \prime}\left(\nu q_{2} e^{r(T-t)}\right)
\end{array}\right),
$$

and

$$
\mathbf{C}=\left(\begin{array}{cc}
M_{X}^{\prime \prime}\left(\nu q_{1} e^{r(T-t)}\right) \cdot M_{Y}\left(\nu q_{2} e^{r(T-t)}\right) & M_{X}^{\prime}\left(\nu q_{1} e^{r(T-t)}\right) \cdot M_{Y}^{\prime}\left(\nu q_{2} e^{r(T-t)}\right) \\
M_{X}^{\prime}\left(\nu q_{1} e^{r(T-t)}\right) \cdot M_{Y}^{\prime}\left(\nu q_{2} e^{r(T-t)}\right) & M_{Y}^{\prime \prime}\left(\nu q_{2} e^{r(T-t)}\right) \cdot M_{X}\left(\nu q_{1} e^{r(T-t)}\right)
\end{array}\right)
$$

Then, the Hessian matrix can be decomposed as

$$
\left(\begin{array}{cc}
\frac{\partial^{2} \tilde{f}\left(q_{1}, q_{2}\right)}{\partial q_{1}^{2}} & \frac{\partial^{2} \tilde{f}\left(q_{1}, q_{2}\right)}{\partial q_{1} \partial q_{2}} \\
\frac{\partial^{2} \tilde{f}\left(q_{1}, q_{2}\right)}{\partial q_{2} \partial q_{1}} & \frac{\partial^{2} \tilde{f}\left(q_{1}, q_{2}\right)}{\partial q_{2}^{2}}
\end{array}\right)=\mathbf{A} \cdot \nu e^{r(T-t)}+(\mathbf{B}+\lambda \cdot \mathbf{C}) \cdot \nu^{2} e^{2 r(T-t)} .
$$

It is easy to see that $\mathbf{B}$ is a nonnegative definite matrix. Furthermore, by the Cauchy-Schwarz inequality, it is not difficult to prove that $\mathbf{A}$ is a positive definite matrix, and that $\mathbf{C}$ is a nonnegative definite matrix. Thus, the Hessian matrix is a positive definite matrix.

Therefore, the minimizer $\left(q_{1}(T-t), q_{2}(T-t)\right)$ of $\tilde{f}\left(q_{1}, q_{2}\right)$ satisfies the following equations

$$
\left\{\begin{array}{l}
a_{1}+\Lambda\left(2\left(1-q_{1}\right) \sigma_{1}^{2}+2\left(1-q_{2}\right) \lambda \mu_{1} \mu_{2}\right)=M_{X}^{\prime}\left(\nu q_{1} e^{r(T-t)}\right)\left(\lambda_{1}+\lambda M_{Y}\left(\nu q_{2} e^{r(T-t)}\right)\right), \\
a_{2}+\Lambda\left(2\left(1-q_{2}\right) \sigma_{2}^{2}+2\left(1-q_{1}\right) \lambda \mu_{1} \mu_{2}\right)=M_{Y}^{\prime}\left(\nu q_{2} e^{r(T-t)}\right)\left(\lambda_{2}+\lambda M_{X}\left(\nu q_{1} e^{r(T-t)}\right)\right) .
\end{array}\right.
$$

Moreover, we have 
Lemma 3.2. For any $t \in[0, T]$, if both $\left(q_{11}, q_{21}\right)$ and $\left(q_{12}, q_{22}\right)$ are the solution to the equations (3.5), then we have $q_{11}=q_{12}$ and $q_{21}=q_{22}$.

Proof: Assume that $q_{11} \neq q_{12}$ or $q_{21} \neq q_{22}$. By Taylor's Theorem, we have

$$
\begin{aligned}
\tilde{f}\left(q_{11}, q_{21}\right)= & \tilde{f}\left(q_{12}, q_{22}\right)+\left(h \frac{\partial}{\partial q_{1}}+k \frac{\partial}{\partial q_{2}}\right) \tilde{f}\left(q_{12}, q_{22}\right) \\
& +\frac{1}{2 !}\left(h \frac{\partial}{\partial q_{1}}+k \frac{\partial}{\partial q_{2}}\right)^{2} \tilde{f}\left(q_{12}+\theta h, q_{22}+\theta k\right) \\
= & \tilde{f}\left(q_{12}, q_{22}\right)+\frac{1}{2} \Delta_{1},
\end{aligned}
$$

where $h=q_{11}-q_{12}, k=q_{21}-q_{22}$ and

$$
\Delta_{1}=\left(h^{2} \frac{\partial^{2}}{\partial q_{1}{ }^{2}}+2 h k \frac{\partial^{2}}{\partial q_{1} \partial q_{2}}+k^{2} \frac{\partial^{2}}{\partial q_{2}^{2}}\right) \tilde{f}\left(q_{12}+\theta h, q_{22}+\theta k\right) .
$$

From Lemma 3.1, we know that the Hessian matrix is a positive definite matrix, and thus

$$
\sqrt{\frac{\partial^{2} \tilde{f}}{\partial q_{1}^{2}} \cdot \frac{\partial^{2} \tilde{f}}{\partial q_{2}^{2}}}>\left|\frac{\partial^{2} \tilde{f}}{\partial q_{1} \partial q_{2}}\right|
$$

Therefore, when $h \neq 0$ and $k \neq 0$,

$$
\Delta_{1} \geq 2|h k| \sqrt{\frac{\partial^{2} \tilde{f}}{\partial q_{1}^{2}} \cdot \frac{\partial^{2} \tilde{f}}{\partial q_{2}^{2}}}+2 h k \frac{\partial^{2} \tilde{f}}{\partial q_{1} \partial q_{2}}>0
$$

which implies that

$$
\tilde{f}\left(q_{11}, q_{21}\right)=\tilde{f}\left(q_{12}, q_{22}\right)+\frac{1}{2} \Delta_{1}>\tilde{f}\left(q_{12}, q_{22}\right) .
$$

Along the same lines, one can obtain

$$
\tilde{f}\left(q_{12}, q_{22}\right)>\tilde{f}\left(q_{11}, q_{21}\right)
$$

which is contrary to (3.6).

For notational convenience, we rewrite equation (3.5) as

$$
\left\{\begin{array}{l}
a_{1}+\Lambda\left(2\left(1-\frac{n}{\nu} e^{-r(T-t)}\right) \sigma_{1}^{2}+2\left(1-\frac{m}{\nu} e^{-r(T-t)}\right) \lambda \mu_{1} \mu_{2}\right)=M_{X}^{\prime}(n)\left(\lambda_{1}+\lambda M_{Y}(m)\right), \\
a_{2}+\Lambda\left(2\left(1-\frac{m}{\nu} e^{-r(T-t)}\right) \sigma_{2}^{2}+2\left(1-\frac{n}{\nu} e^{-r(T-t)}\right) \lambda \mu_{1} \mu_{2}\right)=M_{Y}^{\prime}(m)\left(\lambda_{2}+\lambda M_{X}(n)\right),
\end{array}\right.
$$

where $n=\nu q_{1} e^{r(T-t)}$ and $m=\nu q_{2} e^{r(T-t)}$. To prove the existence and uniqueness of the solution to $(3.7)$, we need the following two more lemmas. 
Lemma 3.3. For any $t \in[0, T]$, there is a unique positive solution to each of the following equations

$$
\lambda \mu_{1} M_{Y}(m)=\lambda \mu_{1}+2 \Lambda \sigma_{1}^{2}+2 \Lambda \lambda \mu_{1} \mu_{2}\left(1-\frac{m}{\nu} e^{-r(T-t)}\right),
$$

and

$$
\left(\lambda_{1}+\lambda\right) M_{X}^{\prime}(n)=a_{1}+2 \Lambda \lambda \mu_{1} \mu_{2}+2 \Lambda \sigma_{1}^{2}\left(1-\frac{n}{\nu} e^{-r(T-t)}\right) .
$$

Proof: We first discuss the equation (3.8). Let

$$
g_{1}(m)=\lambda \mu_{1}+2 \Lambda \sigma_{1}^{2}+2 \Lambda \lambda \mu_{1} \mu_{2}\left(1-\frac{m}{\nu} e^{-r(T-t)}\right),
$$

and

$$
g_{2}(m)=\lambda \mu_{1} M_{Y}(m)
$$

Then, we have

$$
\begin{aligned}
& g_{2}(0)=\lambda \mu_{1} \\
& g_{2}^{\prime}(m)=E\left(Y e^{m Y}\right)>0, \\
& g_{2}^{\prime \prime}(m)=E\left(Y^{2} e^{m Y}\right)>0 .
\end{aligned}
$$

That is, for any $t \in[0, T], g_{2}(m)$ is an increasing convex function with $g_{2}(0)=\lambda \mu_{1}$. Furthermore, $g_{1}(m)$ is a decreasing linear function with

$$
g_{1}(0)=\lambda \mu_{1}+2 \Lambda \sigma_{1}^{2}+2 \Lambda \lambda \mu_{1} \mu_{2}>g_{2}(0)
$$

Therefore, $g_{1}(m)$ and $g_{2}(m)$ have a unique point of intersection at some $m_{1}(t)>0$. That is, equation (3.8) has a unique positive root.

We now consider the equation (3.9). Let

$$
g_{3}(n)=\left(\lambda_{1}+\lambda\right) M_{X}^{\prime}(n)
$$

and

$$
g_{4}(n)=a_{1}+2 \Lambda \lambda \mu_{1} \mu_{2}+2 \Lambda \sigma_{1}^{2}\left(1-\frac{n}{\nu} e^{-r(T-t)}\right)
$$

Then, we have

$$
\begin{aligned}
& g_{3}(0)=a_{1}, \\
& g_{3}^{\prime}(n)=E\left(X^{2} e^{n X}\right)>0, \\
& g_{3}^{\prime \prime}(n)=E\left(X^{3} e^{n X}\right)>0 .
\end{aligned}
$$

That is, for any $t \in[0, T], g_{3}(n)$ is an increasing convex function with $g_{3}(0)=a_{1}$. Furthermore, $g_{4}(n)$ is a decreasing linear function with

$$
g_{4}(0)=a_{1}+2 \Lambda \lambda \mu_{1} \mu_{2}+2 \Lambda \sigma_{1}^{2}>g_{3}(0)
$$


Therefore, $g_{3}(n)$ and $g_{4}(n)$ have a unique point of intersection at some $n_{1}(t)>0$. That is, the equation (3.9) has a unique positive root.

Lemma 3.4. For any $t \in[0, T]$, there is a unique positive solution to each of the following equations

$$
\left(\lambda_{2}+\lambda\right) M_{Y}^{\prime}(m)=a_{2}+2 \Lambda \lambda \mu_{1} \mu_{2}+2 \Lambda\left(1-\frac{m}{\nu} e^{-r(T-t)}\right) \sigma_{2}^{2}
$$

and

$$
\lambda \mu_{2} M_{X}(n)=\lambda \mu_{2}+2 \Lambda \sigma_{2}^{2}+2 \Lambda \lambda \mu_{1} \mu_{2}\left(1-\frac{n}{\nu} e^{-r(T-t)}\right)
$$

Proof: Similar to the proof of Lemma 3.3, one can show that the equation (3.10) ((3.11)) has a unique positive root $m_{2}(t)\left(n_{2}(t)\right)$.

The next lemma states the existence and uniqueness of the solution to the equation (3.7).

Lemma 3.5. Let $m_{1}(t), n_{1}(t), m_{2}(t)$ and $n_{2}(t)$ be the unique positive roots of the equations (3.8), (3.9), (3.10), and (3.11), respectively. If

$$
\left\{\begin{array}{l}
m_{1}(t) \quad>m_{2}(t), \\
n_{1}(t) \quad<n_{2}(t),
\end{array}\right.
$$

or

$$
\left\{\begin{array}{l}
m_{1}(t) \quad<m_{2}(t), \\
n_{1}(t)>n_{2}(t),
\end{array}\right.
$$

hold for any $t \in[0, T]$, then the equation (3.7) has a unique positive root $(\bar{n}(T-t), \bar{m}(T-t))$.

Proof: Let

$$
\begin{aligned}
H_{1}(n, m)= & a_{1}+\Lambda\left(2\left(1-\frac{n}{\nu} e^{-r(T-t)}\right) \sigma_{1}^{2}+2\left(1-\frac{m}{\nu} e^{-r(T-t)}\right) \lambda \mu_{1} \mu_{2}\right) \\
& -M_{X}^{\prime}(n)\left(\lambda_{1}+\lambda M_{Y}(m)\right)
\end{aligned}
$$

and

$$
\begin{aligned}
H_{2}(n, m)= & a_{2}+\Lambda\left(2\left(1-\frac{m}{\nu} e^{-r(T-t)}\right) \sigma_{2}^{2}+2\left(1-\frac{n}{\nu} e^{-r(T-t)}\right) \lambda \mu_{1} \mu_{2}\right) \\
& -M_{Y}^{\prime}(m)\left(\lambda_{2}+\lambda M_{X}(n)\right) .
\end{aligned}
$$

Assume that $H_{1}(n, m)=0$ with $m=f_{1}(n)$, and that $H_{2}(n, m)=0$ with $m=f_{2}(n)$. Differentiating both sides of $H_{1}(n, m)=0$ with respect to $n$ yields

$$
-2 \Lambda\left(\sigma_{1}^{2}+\mu_{1} \mu_{2} f_{1}^{\prime}(n)\right) \frac{1}{\nu} e^{-r(T-t)}=\lambda f_{1}^{\prime}(n) M_{Y}^{\prime}(m) M_{X}^{\prime}(n)+\left(\lambda M_{Y}(m)+\lambda_{1}\right) M_{X}^{\prime \prime}(n),
$$


and thus

$$
f_{1}^{\prime}(n)=-\frac{\left(\lambda M_{Y}(m)+\lambda_{1}\right) M_{X}^{\prime \prime}(n)+2 \Lambda \sigma_{1}^{2} \frac{1}{\nu} e^{-r(T-t)}}{\lambda M_{Y}^{\prime}(m) M_{X}^{\prime}(n)+2 \Lambda \mu_{1} \mu_{2} \frac{1}{\nu} e^{-r(T-t)}}<0 .
$$

Furthermore, it follows from Lemma 3.3 that the equations $H_{1}(0, m)=0$ and $H_{1}(n, 0)=0$ have unique positive solutions $m_{1}(t)$ and $n_{1}(t)$, respectively. Therefore, for any $t \in[0, T]$, function $f_{1}(n)$ is a decreasing function with

$$
\left\{\begin{array}{l}
f_{1}(0)=m_{1}(t)>0 \\
f_{1}^{-1}(0)=n_{1}(t)>0 .
\end{array}\right.
$$

Along the same lines, from $H_{2}(n, m)=0$, we obtain

$$
f_{2}^{\prime}(n)=-\frac{\lambda M_{Y}^{\prime}(m) M_{X}^{\prime}(n)+2 \Lambda \mu_{1} \mu_{2} \frac{1}{\nu} e^{-r(T-t)}}{\left(\lambda M_{X}(n)+\lambda_{2}\right) M_{Y}^{\prime \prime}(m)+2 \Lambda \sigma_{2}^{2} \frac{1}{\nu} e^{-r(T-t)}}<0 .
$$

Using Lemma 3.4, one can show that the equations $H_{2}(0, m)=0$ and $H_{2}(n, 0)=0$ have unique positive solutions $m_{2}(t)$ and $n_{2}(t)$, respectively. Therefore, for any $t \in[0, T]$, the function $f_{2}(n)$ is also a decreasing function with

$$
\begin{cases}f_{2}(0) & =m_{2}(t)>0 \\ f_{2}^{-1}(0) & =n_{2}(t)>0\end{cases}
$$

Note that, in the above equations, $f_{i}^{-1}(i=1,2)$ is the inverse function of $f_{i}, M_{X}^{\prime \prime}(r)=E\left(X^{2} e^{r X}\right)$, and $M_{Y}^{\prime \prime}(r)=E\left(Y^{2} e^{r Y}\right)$.

Therefore, for any $t \in[0, T]$, if the following inequalities

$$
\begin{cases}f_{1}(0) & >f_{2}(0) \\ f_{1}^{-1}(0) & <f_{2}^{-1}(0)\end{cases}
$$

or

$$
\begin{cases}f_{1}(0) & <f_{2}(0) \\ f_{1}^{-1}(0) & <f_{2}^{-1}(0)\end{cases}
$$

hold, the functions $f_{1}(n)$ and $f_{2}(n)$ have at least one point of intersection at some $\bar{n}(T-t)>0$. Then, it follows from Lemma 3.2 that the equation (3.7) has a unique positive root $(\bar{n}(T-t), \bar{m}(T-$ $t)$ ) with $\bar{m}(T-t)=f_{1}(\bar{n}(T-t))=f_{2}(\bar{n}(T-t))$. 
From Lemma 3.5, we get $\nu q_{1}(T-t) e^{r(T-t)}=\bar{n}(T-t)$ and $\nu q_{2}(T-t) e^{r(T-t)}=\bar{m}(T-t)$, and thus

$$
\left\{\begin{array}{l}
q_{1}(T-t)=\frac{\bar{n}(T-t)}{\nu} e^{-r(T-t)}, \\
q_{2}(T-t)=\frac{\bar{m}(T-t)}{\nu} e^{-r(T-t)} .
\end{array}\right.
$$

Assume that $\hat{q}_{1}(T-t)$ and $\hat{q}_{2}(T-t)$ are the unique positive solutions to the following equations:

$$
a_{1}+2 \Lambda\left(1-q_{1}\right) \sigma_{1}^{2}=M_{X}^{\prime}\left(\nu q_{1} e^{r(T-t)}\right)\left(\lambda_{1}+\lambda M_{Y}\left(\nu e^{r(T-t)}\right)\right.
$$

and

$$
a_{2}+2 \Lambda\left(1-q_{2}\right) \sigma_{2}^{2}=M_{Y}^{\prime}\left(\nu q_{2} e^{r(T-t)}\right)\left(\lambda_{2}+\lambda M_{X}\left(\nu e^{r(T-t)}\right)\right.
$$

respectively. Let $t_{1}\left(t_{01}\right)$ and $t_{2}\left(t_{02}\right)$ be the time points at which $q_{1}(T-t)=1\left(\hat{q}_{1}(T-t)=1\right)$ and $q_{2}(T-t)=1\left(\hat{q}_{2}(T-t)=1\right)$, respectively. By the convexity of the function $\tilde{f}$, we derive the optimal reinsurance strategy

$$
\left(q_{1}^{*}(T-t), q_{2}^{*}(T-t)\right)= \begin{cases}\left(q_{1}(T-t), q_{2}(T-t)\right), & 0<t<t_{2}, \\ \left(\hat{q}_{1}(T-t), 1\right), & t_{2} \leq t<t_{01}, \\ (1,1), & t \geq t_{01},\end{cases}
$$

when $t_{1}>t_{2}$, and

$$
\left(q_{1}^{*}(T-t), q_{2}^{*}(T-t)\right)= \begin{cases}\left(q_{1}(T-t), q_{2}(T-t)\right), & 0<t<t_{1}, \\ \left(1, \hat{q}_{2}(T-t)\right), & t_{1} \leq t<t_{02}, \\ (1,1), & t \geq t_{02},\end{cases}
$$

when $t_{1} \leq t_{2}$.

Putting the optimal reinsurance strategies $\left(q_{1}^{*}(T-t), q_{2}^{*}(T-t)\right)$ back into (3.4) yields

$$
h_{1}(T-t)=-\frac{1}{r} c \nu\left(e^{r(T-t)}-1\right)-\left(\lambda_{1}+\lambda_{2}+\lambda\right)(T-t)+\int_{0}^{T-t} K(s) d s,
$$

where

$$
\begin{aligned}
K(s)= & \delta\left(q_{1}^{*}(s), q_{2}^{*}(s)\right) \nu e^{r s}+\lambda_{1} M_{X}\left(\nu q_{1}^{*}(s) e^{s}\right) \\
& +\left(\lambda_{2}+\lambda M_{X}\left(\nu q_{1}^{*}(s) e^{s}\right)\right) M_{Y}\left(\nu q_{2}^{*}(s) e^{s}\right) .
\end{aligned}
$$

To summarize, we have 
Theorem 3.1. Let $\left.\left(q_{1}(T-t), q_{2}(T-t)\right)\right)$ be given in the equation (3.12), and $\hat{q}_{1}(T-t)$ and $\hat{q}_{2}(T-t)$ be the unique positive solution to the equations (3.13) and (3.14), respectively. Then, for any $t \in[0, T]$, the optimal reinsurance strategy for the risk model (2.1) is

$$
\left(q_{1}^{*}(T-t), q_{2}^{*}(T-t)\right)= \begin{cases}\left(q_{1}(T-t), q_{2}(T-t)\right), & 0<t<t_{2}, \\ \left(\hat{q}_{1}(T-t), 1\right), & t_{2} \leq t<t_{01}, \\ (1,1), & t \geq t_{01},\end{cases}
$$

for $t_{1}>t_{2}$, and

$$
\left(q_{1}^{*}(T-t), q_{2}^{*}(T-t)\right)= \begin{cases}\left(q_{1}(T-t), q_{2}(T-t)\right), & 0<t<t_{1}, \\ \left(1, \hat{q}_{2}(T-t)\right), & t_{1} \leq t<t_{02}, \\ (1,1), & t \geq t_{02},\end{cases}
$$

for $t_{1} \leq t_{2}$. Moreover, the value function is given by

$$
V(t, x)=-\frac{m}{\nu} \exp \left\{-\nu x e^{r(T-t)}+h_{1}(T-t)\right\},
$$

where $h_{1}(T-t)$ is defined in (3.15).

Remark 3.1. When the two compound Poisson processes $S_{1}(t)$ and $S_{2}(t)$ have the same distribution, i.e., $\lambda_{1}=\lambda_{2}, \mu_{1}=\mu_{2}$, and $\sigma_{1}=\sigma_{2}$, it is not difficult to see from (3.8)-(3.11) that $m_{1}(t)=n_{2}(t)$ and $n_{1}(t)=m_{2}(t)$. By symmetry, we have $\bar{m}(T-t)=\bar{n}(T-t)$, and therefore $q_{1}^{*}(T-t)=q_{2}^{*}(T-t)$.

\section{Optimal results for the diffusion model}

In this section, we discuss the optimization problem for the diffusion risk model. The surplus process of the risk model (2.2) evolves as

$$
\begin{aligned}
d \hat{R}_{t}^{q_{1}, q_{2}}= & {\left[r \hat{R}_{t}^{q_{1}, q_{2}}+\left(c-\delta\left(q_{1 t}, q_{2 t}\right)\right)-q_{1 t} a_{1}-q_{2 t} a_{2}\right] d t } \\
& +\sqrt{\sigma_{1}^{2} q_{1 t}^{2}+\sigma_{2}^{2} q_{2 t}^{2}+2 q_{1 t} q_{2 t} \lambda \mu_{1} \mu_{2}} d B_{t},
\end{aligned}
$$

and the corresponding HJB equation is

$$
\begin{gathered}
\sup _{q_{1}, q_{2}}\left\{\quad V_{t}+\left[r x+c-\delta\left(q_{1}, q_{2}\right)-q_{1} a_{1}-q_{2} a_{2}\right] V_{x}\right. \\
\left.+\frac{1}{2}\left(\sigma_{1}^{2} q_{1}^{2}+\sigma_{2}^{2} q_{2}^{2}+2 q_{1} q_{2} \lambda \mu_{1} \mu_{2}\right) V_{x x}\right\}
\end{gathered}
$$


for $t<T$, with the boundary condition $V(T, x)=u(x)$.

To derive the optimal reinsurance strategy which satisfies the HJB equation (4.1), we again try to use a solution with the form (3.2). After substituting (3.3) into (4.1) and some algebraic manipulation, we obtain

$$
\begin{aligned}
\inf _{q_{1}, q_{2}}\{\quad & -h^{\prime}(T-t)-\left[c-\delta\left(q_{1}, q_{2}\right)-q_{1} a_{1}-q_{2} a_{2}\right] \nu e^{r(T-t)} \\
& \left.+\frac{1}{2}\left(\sigma_{1}^{2} q_{1}^{2}+\sigma_{2}^{2} q_{2}^{2}+2 q_{1} q_{2} \lambda \mu_{1} \mu_{2}\right) \nu^{2} e^{2 r(T-t)}\right\}=0 .
\end{aligned}
$$

Let

$$
\begin{aligned}
\tilde{f}_{1}\left(q_{1}, q_{2}\right)= & \left(\delta\left(q_{1}, q_{2}\right)+q_{1} a_{1}+q_{2} a_{2}\right) \nu e^{r(T-t)} \\
& +\frac{1}{2}\left(\sigma_{1}^{2} q_{1}^{2}+\sigma_{2}^{2} q_{2}^{2}+2 q_{1} q_{2} \lambda \mu_{1} \mu_{2}\right) \nu^{2} e^{2 r(T-t)} .
\end{aligned}
$$

Then, for any $t \in[0, T]$, we get

$$
\left\{\begin{aligned}
\frac{\partial \tilde{f}_{1}\left(q_{1}, q_{2}\right)}{\partial q_{1}}= & -\left(2 \Lambda\left(\left(1-q_{1}\right) \sigma_{1}^{2}+\left(1-q_{2}\right) \lambda \mu_{1} \mu_{2}\right)\right) \nu e^{r(T-t)} \\
& +\left(q_{1} \sigma_{1}^{2}+\lambda q_{2} \mu_{1} \mu_{2}\right) \nu^{2} e^{2 r(T-t)}, \\
\frac{\partial^{2} \tilde{f}_{1}\left(q_{1}, q_{2}\right)}{\partial q_{1}^{2}}= & 2 \Lambda \sigma_{1}^{2} \nu e^{r(T-t)}+\sigma_{1}^{2} \nu^{2} e^{2 r(T-t)}>0, \\
\frac{\partial \tilde{f}_{1}\left(q_{1}, q_{2}\right)}{\partial q_{2}}= & -\left(2 \Lambda\left(\left(1-q_{2}\right) \sigma_{2}^{2}+\left(1-q_{1}\right) \lambda \mu_{1} \mu_{2}\right)\right) \nu e^{r(T-t)} \\
& +\left(q_{2} \sigma_{2}^{2}+\lambda q_{1} \mu_{1} \mu_{2}\right) \nu^{2} e^{2 r(T-t)}, \\
\frac{\partial^{2} \tilde{f}_{1}\left(q_{1}, q_{2}\right)}{\partial q_{2}^{2}}= & 2 \Lambda \sigma_{2}^{2} \nu e^{r(T-t)}+\sigma_{2}^{2} \nu^{2} e^{2 r(T-t)}>0, \\
\frac{\partial^{2} \tilde{f}_{1}\left(q_{1}, q_{2}\right)}{\partial q_{1} \partial q_{2}}= & 2 \Lambda \lambda \mu_{1} \mu_{2} \nu e^{r(T-t)}+\lambda \mu_{1} \mu_{2} \nu^{2} e^{2 r(T-t)} .
\end{aligned}\right.
$$

The Hessian matrix in this case is given by $\mathbf{A} \cdot\left(2 \Lambda+\nu e^{r(T-t)}\right)$, which is also a positive definite matrix. Thus, $\tilde{f}_{1}\left(q_{1}, q_{2}\right)$ is a convex function with respect to $q_{1}$ and $q_{2}$. Therefore, the minimizer $\left(\bar{q}_{1}(T-t), \bar{q}_{2}(T-t)\right)$ of $\tilde{f}_{1}\left(q_{1}, q_{2}\right)$ satisfies the following equations

$$
\left\{\begin{array}{l}
-\left(2 \Lambda\left(\left(1-q_{1}\right) \sigma_{1}^{2}+\left(1-q_{2}\right) \lambda \mu_{1} \mu_{2}\right)\right)+\left(q_{1} \sigma_{1}^{2}+\lambda q_{2} \mu_{1} \mu_{2}\right) \nu e^{r(T-t)}=0 \\
-\left(2 \Lambda\left(\left(1-q_{2}\right) \sigma_{2}^{2}+\left(1-q_{1}\right) \lambda \mu_{1} \mu_{2}\right)\right)+\left(q_{2} \sigma_{2}^{2}+\lambda q_{1} \mu_{1} \mu_{2}\right) \nu e^{r(T-t)}=0
\end{array}\right.
$$

which can be rewritten as

$$
\left\{\begin{aligned}
q_{1} \sigma_{1}^{2}+\lambda q_{2} \mu_{1} \mu_{2} & =\frac{2 \Lambda \sigma_{1}^{2}+2 \Lambda \lambda \mu_{1} \mu_{2}}{2 \Lambda+\nu e^{r(T-t)}} \\
q_{2} \sigma_{2}^{2}+\lambda q_{1} \mu_{1} \mu_{2} & =\frac{2 \Lambda \sigma_{2}^{2}+2 \Lambda \lambda \mu_{1} \mu_{2}}{2 \Lambda+\nu e^{r(T-t)}}
\end{aligned}\right.
$$


Then, it is not difficult to derive the following solution

$$
\left\{\begin{aligned}
\bar{q}_{1}(T-t) & =\frac{2 \Lambda}{2 \Lambda+\nu e^{r(T-t)}} \\
\bar{q}_{2}(T-t) & =\frac{2 \Lambda}{2 \Lambda+\nu e^{r(T-t)}}
\end{aligned}\right.
$$

Since

$$
\frac{2 \Lambda}{2 \Lambda+\nu e^{r(T-t)}} \in(0,1)
$$

we obtain the optimal reinsurance strategy

$$
q_{1 t}^{*}=q_{2 t}^{*}=\frac{2 \Lambda}{2 \Lambda+\nu e^{r(T-t)}} .
$$

Putting $q_{1 t}^{*}=q_{2 t}^{*}=\bar{q}_{1}(T-t)$ into (4.2) yields

$$
h_{3}(T-t)=\frac{1}{r}\left(a_{0}-c\right) \nu\left(e^{r(T-t)}-1\right)+\int_{0}^{T-t} \bar{K}_{1}(s) d s,
$$

with

$$
\bar{K}_{1}(s)=\Lambda\left(1-\bar{q}_{1}(s)\right) \sigma_{0}^{2} \nu e^{r s}+\frac{1}{2} \bar{q}_{1}(s)^{2} \sigma_{0}^{2} \nu^{2} e^{2 r s} .
$$

Finally, we summarize the result of this subsection in the following theorem.

Theorem 4.1. For any $t \in[0, T]$, the optimal reinsurance strategy for the risk model (2.2) is

$$
q_{1 t}^{*}=q_{2 t}^{*}=\frac{2 \Lambda}{2 \Lambda+\nu e^{r(T-t)}},
$$

and the value function is

$$
V(t, x)=-\frac{m}{\nu} \exp \left\{-\nu x e^{r(T-t)}+h_{3}(T-t)\right\} .
$$

where $h_{3}(T-t)$ is defined in (4.3).

Remark 4.1. From (4.4), we see that the optimal reinsurance strategy in this case is the same as the one in Theorems 4.1 and 5.2 of Liang et al. (2011).

Remark 4.2. The optimal reinsurance strategies for the diffusion model depends only on the safety loading $\Lambda$, time $T-t$, and interest rate $r$. That is, the claim size distributions as well as the counting processes have no effect on the optimal reinsurance strategy. However, from the numerical results shown in Section 5, the optimal results for the compound Poisson model depend not only on the safety loading $\Lambda$, time $T-t$, and interest rate $r$, but also on the claim size distributions and the counting processes. 


\section{Numerical examples}

In this section, we assume that the claim sizes $X_{i}$ and $Y_{i}$ are exponentially distributed with parameters $\alpha_{1}$ and $\alpha_{2}$, respectively. Then, we have

$$
\left\{\begin{aligned}
M_{X}\left(\nu q_{1} e^{r(T-t)}\right) & =\frac{\alpha_{1}}{\alpha_{1}-\nu q_{1} e^{r(T-t)}} \\
M_{Y}\left(\nu q_{2} e^{r(T-t)}\right) & =\frac{\alpha_{2}}{\alpha_{2}-\nu q_{2} e^{r(T-t)}}
\end{aligned}\right.
$$

The minimizer $\left(q_{1}(T-t), q_{2}(T-t)\right)$ of $(3.3)$ satisfies the following equations

$$
\left\{\begin{aligned}
a_{1}+\Lambda\left(2\left(1-q_{1}\right) \sigma_{1}^{2}+2\left(1-q_{2}\right) \lambda \mu_{1} \mu_{2}\right) & =\frac{\alpha_{1}}{\left(\alpha_{1}-\nu q_{1} e^{r(T-t)}\right)^{2}}\left(\lambda_{1}+\frac{\lambda \alpha_{2}}{\alpha_{2}-\nu q_{2} e^{r(T-t)}}\right) \\
a_{2}+\Lambda\left(2\left(1-q_{2}\right) \sigma_{2}^{2}+2\left(1-q_{1}\right) \lambda \mu_{1} \mu_{2}\right) & =\frac{\alpha_{2}}{\left(\alpha_{2}-\nu q_{2} e^{r(T-t)}\right)^{2}}\left(\lambda_{2}+\frac{\lambda \alpha_{1}}{\alpha_{1}-\nu q_{1} e^{r(T-t)}}\right)
\end{aligned}\right.
$$

with $\mu_{1}=1 / \alpha_{1}, \mu_{2}=1 / \alpha_{2}, \sigma_{1}^{2}=2\left(\lambda_{1}+\lambda\right) / \alpha_{1}^{2}$ and $\sigma_{2}^{2}=2\left(\lambda_{2}+\lambda\right) / \alpha_{2}^{2}$.

Example 5.1. In this example, we set $\lambda_{1}=3, r=0.05, T=10, \lambda_{2}=4, \Lambda=2, \lambda=2, \alpha_{1}=2$, and $\alpha_{2}=3$. The results are shown in Tables 5.1, 5.2 and 5.3.

Table 5.1 The effect of $t$ on the optimal reinsurance strategies

\begin{tabular}{ccccccccc}
\hline \hline$t$ & 1 & 2 & 3 & 4 & 5 & 6 & 7 & 8 \\
\hline$q_{1}^{*}$ & 0.73625 & 0.75017 & 0.76353 & 0.77632 & 0.78854 & 0.80019 & 0.81127 & 0.82178 \\
$q_{2}^{*}$ & 0.77252 & 0.78397 & 0.79492 & 0.80540 & 0.81540 & 0.82493 & 0.83401 & 0.84265 \\
\hline
\end{tabular}

Table 5.2 The effect of $\nu$ on the optimal reinsurance strategies

\begin{tabular}{ccccccccc}
\hline \hline$\nu$ & 0.2 & 0.3 & 0.4 & 0.5 & 0.6 & 0.7 & 0.8 & 0.9 \\
\hline$q_{1}^{*}$ & 0.92604 & 0.88254 & 0.83618 & 0.78854 & 0.74123 & 0.69558 & 0.65249 & 0.61245 \\
$q_{2}^{*}$ & 0.93083 & 0.89324 & 0.85452 & 0.81540 & 0.77663 & 0.73883 & 0.70249 & 0.66794 \\
\hline
\end{tabular}

Table 5.3 The effect of $\Lambda$ on the optimal reinsurance strategies

\begin{tabular}{cccccccccc}
\hline \hline$\Lambda$ & 1 & 2 & 3 & 4 & 5 & 6 & 8 & 9 & 10 \\
\hline$q_{1}^{*}$ & 0.87869 & 0.78854 & 0.84280 & 0.87453 & 0.89547 & 0.91037 & 0.93019 & 0.93818 & 0.94472 \\
$q_{2}^{*}$ & 0.67927 & 0.81540 & 0.86604 & 0.89473 & 0.91325 & 0.92620 & 0.94316 & 0.94109 & 0.93940 \\
\hline
\end{tabular}


From Table 5.1 with $\nu=0.5$ and $\Lambda=2$, we see that the optimal reinsurance strategies increase as $t$ increases. Note that $\nu$ is the constant absolute risk aversion parameter of the utility function, a large value of $\nu$ means more risk averse. In Table 5.2 with $t=5$ and $\Lambda=2$, we observe that the optimal reinsurance strategies decrease as $\nu$ increases. This implies that if the decision maker is more risk averse, a larger portion of the underlying risk will be transferred to a reinsurer. Besides, the results in Table 5.3 with $t=5$ and $\nu=0.5$ indicate that the optimal reinsurance strategies do not necessarily increase as the value of $\Lambda$ increases.

Example 5.2. In this example, we put $\lambda_{1}=3, r=0.05, T=10, \lambda_{2}=4, \nu=0.5, \lambda=2$, $t=5$, and $\Lambda=2$. Tables 5.4 and 5.5 present the impact of $\alpha_{1}$ and $\alpha_{2}$ on the optimal reinsurance strategies.

Table 5.4 The effect of $\alpha_{1}$ on the optimal reinsurance strategies

\begin{tabular}{cccccccccc}
\hline \hline$\alpha_{1}$ & 1 & 2 & 3 & 4 & 5 & 6 & 7 & 9 & 10 \\
\hline$q_{1}^{*}$ & 0.69452 & 0.78854 & 0.81495 & 0.82740 & 0.83487 & 0.84003 & 0.84392 & 0.84968 & 0.85197 \\
$q_{2}^{*}$ & 0.82599 & 0.81540 & 0.81647 & 0.81755 & 0.81832 & 0.81889 & 0.81931 & 0.81990 & 0.82012 \\
\hline
\end{tabular}

Table 5.5 The effect of $\alpha_{2}$ on the optimal reinsurance strategies

\begin{tabular}{cccccccccc}
\hline \hline$\alpha_{2}$ & 0.9 & 1 & 2 & 3 & 4 & 5 & 6 & 7 & 9 \\
\hline$q_{1}^{*}$ & 0.78598 & 0.78378 & 0.78512 & 0.78854 & 0.79057 & 0.79185 & 0.79273 & 0.79337 & 0.79423 \\
$q_{2}^{*}$ & 0.66971 & 0.69245 & 0.78767 & 0.81540 & 0.82911 & 0.83781 & 0.84417 & 0.84926 & 0.85739 \\
\hline
\end{tabular}

We see from Table 5.4 with $\alpha_{2}=3$ and Table 5.5 with $\alpha_{1}=2$ that a greater value of $\alpha_{i}$ yields a greater value of the optimal reinsurance strategy $q_{i}^{*}$. However, $q_{1}^{*}\left(q_{2}^{*}\right)$ does not necessarily increase as the value of $\alpha_{2}\left(\alpha_{1}\right)$ increases. We can also observe from Tables 5.4 and 5.5 that the value of $q_{1}^{*}$ is always smaller than the value of $q_{2}^{*}$ when the inequality $\alpha_{1}<\alpha_{2}$ holds, and vice versa. This implies that the values of the optimal reinsurance strategies are more sensitive to the change in the claim size distributions than to the change in the counting processes (see also Table 5.7). 
Example 5.3. For $r=0.05, T=10, \nu=0.5, \Lambda=2, t=5, \alpha_{1}=2$, and $\alpha_{2}=3$, the optimal results are displayed in Tables 5.6 and 5.7.

Table 5.6 The effect of $\lambda$ on the optimal reinsurance strategies

\begin{tabular}{cccccccccc}
\hline \hline$\lambda$ & 1 & 2 & 3 & 4 & 5 & 6 & 7 & 8 & 9 \\
\hline$q_{1}^{*}$ & 0.79189 & 0.78854 & 0.78643 & 0.78495 & 0.78387 & 0.78303 & 0.78236 & 0.78181 & 0.78135 \\
$q_{2}^{*}$ & 0.81770 & 0.81540 & 0.81413 & 0.81335 & 0.81284 & 0.81248 & 0.81223 & 0.81205 & 0.81191 \\
\hline
\end{tabular}

Table 5.7 The effect of $\lambda_{2}$ on the optimal reinsurance strategies

\begin{tabular}{cccccccccc}
\hline \hline$\lambda_{2}$ & 2 & 4 & 5 & 8 & 10 & 12 & 14 & 16 & 18 \\
\hline$q_{1}^{*}$ & 0.78904 & 0.78854 & 0.78840 & 0.78815 & 0.78806 & 0.78799 & 0.78794 & 0.78790 & 0.78786 \\
$q_{2}^{*}$ & 0.81171 & 0.81540 & 0.81644 & 0.81832 & 0.81904 & 0.81956 & 0.81994 & 0.82024 & 0.82048 \\
\hline
\end{tabular}

Table 5.6 with $\lambda_{1}=3$ and $\lambda_{2}=4$ shows that the optimal reinsurance strategies decrease while the value of $\lambda$ increases. This is consistent with the fact that the insurer would rather retain a less share of each claim when the expected claim number becomes larger. On the other hand, the numerical values in Table 5.7 with $\lambda=2$ and $\lambda_{1}=3$ indicate that a greater value of $\lambda_{2}$ yields a greater value of $q_{2}^{*}$ but a smaller value of $q_{1}^{*}$. Along the same lines, one can numerically show that a greater value of $\lambda_{1}$ yields a greater value of $q_{1}^{*}$ but a smaller value of $q_{2}^{*}$. Finally, Tables 5.6 and 5.7 also exhibit that the changes in the optimal reinsurance strategies are small. These suggest that the optimal reinsurance strategies are kind of insensitive to the change in the counting processes.

\section{Conclusion}

We first recap the main results of the paper. From an insurer's point of view, we consider the optimal proportional reinsurance strategy in a risk model with two dependent classes of insurance business, where the two claim number processes are correlated. By a nonstandard approach, we investigate the existence and uniqueness of the optimal reinsurance strategy. Under the criterion of maximizing the expected exponential utility together with the variance premium principle, closedform expressions for the optimal strategy and value function are given not only for the compound 
Poisson risk model but also for the diffusion model. Furthermore, we find that the optimal reinsurance strategies in the diffusion risk model only depend on the safety loading, time, and interest rate. However, from the numerical examples, we see that the optimal results for the compound Poisson model depend not only on the safety loading, time, and interest rate, but also on the claim size distributions and the counting processes.

In this paper, we may extend our work to the case of Centeno (2005) in which different safety loadings are used for the two classes of insurance business. In this case, the reinsurance premium rate becomes

$$
\delta\left(q_{1}, q_{2}\right)=\left(1-q_{1}\right) a_{1}+\Lambda_{1}\left(1-q_{1}\right)^{2} \sigma_{1}^{2}+\left(1-q_{2}\right) a_{2}+\Lambda_{2}\left(1-q_{2}\right)^{2} \sigma_{2}^{2}
$$

Since the dependence between $S_{1}(t)\left(\hat{S}_{1}(t)\right)$ and $S_{2}(t)\left(\hat{S}_{2}(t)\right)$ is not considered in $(6.1)$, it is simpler than (3.1) and results in a simpler version of the equation (3.5). Moreover, following the ideas and steps in Section 3, one can investigate the existence and uniqueness of the optimal reinsurance strategies, and derive closed form expressions for the optimal results for the compound Poisson risk model. On the other hand, it can be shown that the optimal reinsurance strategies for the diffusion risk model are given by

$$
\left\{\begin{aligned}
q_{1 t}^{*} & =\frac{2 \Lambda_{1} \sigma_{1}^{2} \sigma_{2}^{2}\left(2 \Lambda_{2}+\nu e^{r(T-t)}\right)-2 \Lambda_{2} \sigma_{2}^{2} \lambda \mu_{1} \mu_{2} \nu e^{r(T-t)}}{\sigma_{1}^{1} \sigma_{2}^{2}\left(2 \Lambda_{2}+\nu e^{r(T-t)}\right)\left(2 \Lambda_{1}+\nu e^{r(T-t)}\right)-\lambda^{2} \mu_{1}^{2} \mu_{2}^{2} \nu^{2} e^{2 r(T-t)}}, \\
q_{2 t}^{*} & =\frac{2 \Lambda_{2} \sigma_{1}^{2} \sigma_{2}^{2}\left(2 \Lambda_{1}+\nu e^{r(T-t)}\right)-2 \Lambda_{1} \sigma_{1}^{2} \lambda \mu_{1} \mu_{2} \nu e^{r(T-t)}}{\sigma_{1}^{1} \sigma_{2}^{2}\left(2 \Lambda_{2}+\nu e^{r(T-t)}\right)\left(2 \Lambda_{1}+\nu e^{r(T-t)}\right)-\lambda^{2} \mu_{1}^{2} \mu_{2}^{2} \nu^{2} e^{2 r(T-t)}} .
\end{aligned}\right.
$$

It is obvious that the optimal reinsurance strategies are different in the diffusion risk model. They depend not only on the safety loading, time, and interest rate, but also on the claim size distributions and the counting processes.

Although the literature on optimal reinsurance is increasing rapidly, very few of these contributions deal with the problem in relation to dependent risks. Therefore, there are still some interesting problems in this direction that can be further studied. For example, one may consider the optimal reinsurance with dependent risks under the partial information, multi-criteria, or stochastic differential games. Moreover, the optimal reinsurance problem with expected utility under additional constraints on the probability of ruin is a very challenging problem, especially for risk processes with jumps.

\section{Acknowledgements}


The authors would like to thank the anonymous referee for his/her careful reading and helpful comments on an earlier version of this paper, which led to a considerable improvement of the presentation of the work. The authors also give sincere thanks to Dr. Jinli Sun, a colleague of Dr. Zhibin Liang, who gives a helpful comments on the proof of Lemma 3.2. The research of Zhibin Liang was supported by the National Natural Science Foundation of China (Grant No.11101215). The research of Kam Chuen Yuen was supported by a grant from the Research Grants Council of the Hong Kong Special Administrative Region, China (Project No. HKU 7057/13P), and the CAE 2013 research grant from the Society of Actuaries - any opinions, finding, and conclusions or recommendations expressed in this material are those of the authors and do not necessarily reflect the views of the SOA.

\section{References}

[1] Bai, L. Cai, J. and Zhou, M. (2012). Optimal dynamic risk control strategies for an insurer with dependent risks. Working paper.

[2] Bernard, C. and Tian, W. (2009). Optimal reinsurance arrangements under tail risk measures. Journal of Risk and Insurance 76(3), 709-725.

[3] Browne, S. (1995). Optimal investment policies for a firm with random risk process: exponential utility and minimizing the probability of ruin. Mathematics of Operations Research 20, 937-958.

[4] Cai, J. and Tan, K. (2007). Optimal retention for a stop-loss reinsurance under the VaR and CTE risk measures. ASTIN Bulletin 37(1), 93-112.

[5] Cai, J. Tan, K. Weng, C. and Zhang, Y. (2008). Optimal reinsurance under VaR and CTE risk measures. Insurance: Mathematics and Economics 43, 185-196.

[6] Centeno, M. (1986). Measuring the effects of reinsurance by the adjustment coefficient. Insurance: Mathematics and Economics 5, 169-182.

[7] Centeno, M. (2002). Excess of loss reinsurance and Gerber's inequality in the Sparre Anderson model. Insurance: Mathematics and Economics 31, 415-427.

[8] Centeno, M. (2005). Dependent risks and excess of loss reinsurance. Insurance: Mathematics and Economics 37, 229-238.

[9] Fleming, W. and Soner, H. (2006). Controlled Markov Processes and Viscosity Solutions. 2nd Ed. Springer-Verlag, New York.

[10] Gerber, H. (1979). An Introduction to Mathematical Risk Theory. In: S.S. Huebner Foundation Monograph, Series No. 8. Irwin, Homewood, Ill. 
[11] Grandell, J. (1991). Aspects of Risk Theory. Springer-Verlag, New York.

[12] Hald, M. and Schmidli, H. (2004). On the maximization of the adjustment coefficient under proportioal reinsurance. ASTIN Bulletin 34, 75-83.

[13] Hipp, C. and Taksar, M. (2010). Optimal non-proportional reinsurance control. Insurance: Mathematics and Economics 47, 246-254.

[14] Irgens, C. and Paulsen, J. (2004). Optimal control of risk exposure, reinsurance and investments for insurance portfolios. Insurance: Mathematics and Economics 35, 21-51.

[15] Kaluszka, M. (2001). Optimal reinsurance under mean-variance premium principles. Insurance: Mathematics and Economics 28, 61-67.

[16] Kaluszka, M. (2004). Mean-variance optimal reinsurance arrangements. Scandinavian Actuarial Journal, $28-41$.

[17] Liang, Z. (2007). Optimal proportional reinsurance for controlled risk process which is perturbed by diffusion. Acta Mathematicae Applicatae Sinica, English Series 23, 477-488.

[18] Liang, Z. and Guo, J. (2007). Optimal proportional reinsurance and ruin probability. Stochastic Models $23,333-350$.

[19] Liang, Z. and Guo, J. (2008). Upper bound for ruin probabilities under optimal investment and proportional reinsurance. Applied Stochastic Models in Business and Industry 24, 109-128.

[20] Liang, Z. and Young, V. (2012). Dividends and reinsurance under a penalty for ruin. Insurance: Mathematics and Economics 50, 437-445.

[21] Liang, Z. Yuen, K.C. and Cheung, K.C. (2012). Optimal reinsurance-investment problem in a constant elasticity of variance tock market for jump-diffusion risk model. Applied Stochastic Models in Business and Industry 28, 585-597.

[22] Liang, Z. Yuen, Kam C. and Guo, J. (2011). Optimal proportional reinsurance and investment in a stock market with Ornstein-Uhlenbeck process. Insuarnce: Mathematics and Economics 49, 207-215.

[23] Luo, S. Taksar, M. and Tsoi, A. (2008). On reinsurance and investment for large insurance portfolios. Insurance: Mathematics and Economics 42, 434-444.

[24] Promislow, D. and Young, V. (2005). Minimizing the probability of ruin when claims follow Brownian motion with drift. North American Actuarial Journal 9(3), 109-128.

[25] Schmidli, H. (2001). Optimal proportional reinsurance policies in a dynamic setting. Scandinavian Actuarial Journal, 55-68.

[26] Schmidli, H. (2002). On minimizing the ruin probability by investment and reinsurance. Annals of Applied Probability 12, 890-907.

[27] Wang, G. and Yuen, Kam C. (2005). On a correlated aggregate claims model with thinning-dependence structure. Insurance: Mathematics and Economics 36, 456-468. 
[28] Yang, H. and Zhang, L. (2005). Optimal investment for insurer with jump-diffusion risk process. Insurance: Mathematics and Economics 37, 615-634.

[29] Yuen, Kam C. Guo, J. and Wu, X. (2002). On a correclated aggregate claim model with Poisson and Erlang risk process. Insurance: Mathematics and Economics 31, 205-214.

[30] Yuen, Kam C. Guo, J. and Wu, X. (2006). On the first time of ruin in the bivariate compound Poisson model. Insurance: Mathematics and Economics 38, 298-308.

Correspondence author: Dr. Zhibin Liang, School of Mathematical Sciences, Nanjing Normal University, Jiangsu 210023, P.R.China 